\title{
Non-perturbative effects of the electroweak interaction at the LHC
}

\author{
B.A. Arbuzov ${ }^{1, \star}$ \\ ${ }^{1}$ M.V. Lomonosov Moscow State University, 119991 Moscow, Russia
}

\begin{abstract}
Assuming an existence of the anomalous triple electro-weak bosons interaction being defined by coupling constant $\lambda$ we calculate its contribution to interactions of the Higgs with pairs of heavy particles. Bearing in mind experimental restrictions $-0.011<\lambda<0.011$ we present results for possible effects in processes $p p \rightarrow W^{+} W^{-} H, p p \rightarrow W^{+} Z H, p p \rightarrow W^{-} Z H, p p \rightarrow \bar{t} t H, p p \rightarrow \bar{b} b H$. Effects could be significant with negative sign of $\lambda$ in associated heavy quarks $t, b$ pairs production with the Higgs. In calculations we rely on results of the non-perturbative approach to a spontaneous generation of effective interactions, which defines the form-factor of the three-boson anomalous interaction.
\end{abstract}

\section{Introduction}

The totality of data nowadays confirms main features of the Standard Model, which consists of QCD, describing strong interactions, and the EW theory, describing electroweak interactions. This confirmation is essentially based on numerous perturbative calculations which describe corresponding data. However in QCD the inevitable introduction of non-perturbative effects is also evident. First of all the low momenta region of the strong interaction definitely can not be described in the framework of the perturbative calculations. Examples of non-perturbative quantities are well-known: vacuum averages the gluon condensate $<\frac{\alpha_{s}}{\pi} G_{\mu \nu}^{a} G_{\mu \nu}^{a}>$, the quark condensate $<\bar{q} q>$ etc. One of the most powerful methods of dealing with the non-perturbative effects is provided in the framework of approaches using the so-called effective interactions. The eldest and the most popular such effective interaction is the famous Nambu-Jona-Lasinio interaction [1,2]. With application to quark structure of hadrons this approach adequately describes the low momenta region, see e.g. reviews [3-5].

The Nambu-Jona-Lasinio interaction deals with four-quark effective terms. However, the nonzero gluon condensate testifies for additional effective terms also in gluon interactions. There were also proposals for such terms. In particular, triple gluon interaction in the low momenta region of the following form

$$
L_{e f f}=-\frac{G}{3 !} f_{a b c} G_{\mu \nu}^{a} G_{v \rho}^{b} G_{\rho \mu}^{c} ;
$$

where $G_{\mu \nu}^{a}$ is a gauge covariant gluon field and $f_{a b c}$ are structure constants of the color $S U(3)$, was proposed in work [6].

^e-mail: arbuzov@theory.sinp.msu.ru 
In the electro-weak theory necessity of non-perturbative contribution is not nowadays so evident as in QCD. However the structure of gauge theories is the same for both cases. One might expect similar features in three-boson interactions. In particular, the following three weak boson interaction was introduced $[7,8]$

$$
L_{e f f}=-\frac{G_{W}}{3 !} F\left(p_{i}\right) \epsilon_{a b c} W_{\mu \nu}^{a} W_{\nu \rho}^{b} W_{\rho \mu}^{c} ; \quad G_{W}=\frac{g \lambda}{M_{W}^{2}} ; W_{\mu \nu}^{3}=\cos \theta_{W} Z_{\mu \nu}+\sin \theta_{W} A_{\mu \nu} ;
$$

where $g$ is the electro-weak gauge coupling and indices $a, b, c$ take three values and the third boson $W_{\mu \nu}^{3}$ is a composition of neutral bosons $Z$ and $\gamma$. Form-factor $F\left(p_{i}\right)$ in $[7,8]$ is postulated and it has to vanish for $\left|p_{i}^{2}\right| \gg \Lambda^{2}$, where $\Lambda$ is a characteristic scale. Interaction (2) would lead to effects e.g. in electro-weak bosons pair production and was studied in experiments. The best limitations for parameter $\lambda$ is provided by recent data of the CMS Collaboration [9]

$$
-0.011<\lambda<0.011 \text {. }
$$

The ATLAS Collaboration presents similar results [10]

$$
-0.013<\lambda<0.013 \text {. }
$$

Both the Nambu-Jona-Lasinio interaction and interaction (1) are supposed to act in a low momenta region. This means, that in both cases form-factors are present, which guarantee decreasing of intensity of the interactions for large momenta. In the original NJL [1,2] interaction a cut-off was introduced for the purpose. Starting of fundamental gauge theories of interactions of the Standard Model we have to understand the origin of such effective cut-off. This can be done under assumption of these interactions being spontaneously generated. The notion of spontaneous generation is traced back to methods of the superconductivity theory. In application to superconductivity the conception of compensation principle was elaborated $[11,12]$ by N.N. Bogoliubov. This approach was applied to spontaneous generation of Nambu-Jona-Lasinio interaction in work [13] and of interaction (1) in work [14]. In the course of this application form-factors inherent to corresponding interactions are uniquely defined ${ }^{1}$. As an additional confirmation of applicability of the method to non-perturbative quantities, value $V_{2}$ of the gluon condensate was calculated [14] in agreement with its phenomenological value.

\section{Additional interactions of the Higgs with electro-weak bosons}

Electro-weak bosons $W^{ \pm}, Z$ due to their large masses interact with Higgs $H$ significantly. Namely there are the following vertices for interaction of Higgs $H$ with $W^{+} W^{-}$and $Z Z$ respectively

$$
\iota g M_{W} g_{\mu \nu} ; \quad l \frac{g M_{Z}}{\cos \theta_{W}} g_{\mu \nu} .
$$

Now let us assume, that in addition to usual three-boson gauge interaction, interaction (2) really exists.

Then three-boson vertex takes the form

$$
\begin{aligned}
& V(\mu, p ; v, q ; \rho, k)=-g \epsilon_{a b c}\left(g_{\mu \nu}\left(q_{\rho}-p_{\rho}\right)+g_{v \rho}\left(k_{\mu}-q_{\mu}\right)+g_{\rho \mu}\left(p_{v}-k_{v}\right)+\frac{\lambda}{M_{W}^{2}} F(p, q, k) \times\right. \\
& \left.\left(g_{\mu v}\left(q_{\rho} p k-p_{\rho} q k\right)+g_{v \rho}\left(k_{\mu} p q-q_{\mu} p k\right)+g_{\rho \mu}\left(p_{v} q k-k_{v} p q\right)+q_{\mu} k_{v} p_{\rho}-k_{\mu} p_{v} q_{\rho}\right)\right) .
\end{aligned}
$$

\footnotetext{
${ }^{1}$ Of course, in the framework of an approximation.
} 
Here $F(p, q, k)$ is a form-factor, which is defined in the framework of the spontaneous generation of effective interaction (2) [15] in the compensation approach, which we have discussed in the Introduction. Then we have additional contribution to $V V H$ vertex due to terms proportional to $\lambda$ and to $\lambda^{2}$ according to diagrams presented in Fig. 1. As a consequence we have additional contribution to vertices $V_{V V^{\prime} H}$, where $V$ and $V^{\prime}$ correspond to electro-weak bosons $W, Z, \gamma$.

The combined account of both vertices $(5,6)$ leads to corrections for $V V^{\prime} H$ vertex according to diagrams shown in Fig. 1.

We have for vertices of interactions of the Higgs with two electro-weak bosons $V(p, \mu) V^{\prime}(q, v)$ instead of (5)

$$
\begin{aligned}
& V_{W^{+} W^{-} H}=l g M_{W}\left(g_{\mu \nu}+G_{W W}\left(g_{\mu \nu} p q-p_{\nu} q_{\mu}\right)\right) ; \\
& V_{Z Z H}=\imath \frac{g M_{Z}}{\cos \theta_{W}}\left(g_{\mu \nu}+G_{Z Z}\left(g_{\mu \nu} p q-p_{\nu} q_{\mu}\right)\right) ; \\
& V_{Z A H}=\imath \frac{g M_{Z}}{\cos \theta_{W}} G_{Z \gamma}\left(g_{\mu \nu} p q-p_{\nu} q_{\mu}\right) ; \quad V_{A A H}=\imath \frac{g M_{Z}}{\cos \theta_{W}} G_{\gamma \gamma}\left(g_{\mu \nu} p q-p_{\nu} q_{\mu}\right) ; \\
& G_{Z Z}=\cos ^{2} \theta_{W} G_{W_{0} W_{0}} ; \quad G_{\gamma \gamma}=\sin ^{2} \theta_{W} G_{W_{0} W_{0}} ; \quad G_{Z \gamma}=2 \cos \theta_{W} \sin \theta_{W} G_{W_{0} W_{0}} ; \\
& G_{W_{0} W_{0}}=\frac{2 g}{M_{W}^{2}}\left(\frac{3 g \lambda}{8 \pi^{2}} I_{1}-\frac{\sqrt{2 \lambda^{2}}}{\pi} I_{2}\right) ; \\
& G_{W W}=\frac{g}{M_{W}^{2}}\left(\frac{3 g \lambda}{8 \pi^{2}} I_{W 1}-\frac{\sqrt{2 \lambda^{2}}}{\pi} I_{W 2}+\frac{3 g \lambda}{8 \pi^{2}} I_{Z 1}-\frac{\sqrt{2 \lambda^{2}}}{\pi} I_{Z 2}\right) .
\end{aligned}
$$

Here

$$
\begin{aligned}
& I_{1}=\int_{0}^{z_{0}} \frac{F(t) d t}{2(\sqrt{t}+\mu)^{2}} ; I_{2}=\int_{0}^{z_{0}} \frac{2 t F^{2}(t) d t}{(\sqrt{t}+\mu)^{3}} ; \quad I_{W 1}=\int_{0}^{z_{0}} \frac{F(t)\left(\sqrt{t}+s \mu_{Z}\right) d t}{\left(\sqrt{t}+\mu_{Z}\right)^{2}(\sqrt{t}+\mu)} \\
& I_{W 2}=\int_{0}^{z_{0}} \frac{F^{2}(t) \sqrt{t}\left(\sqrt{t}+s \mu_{Z}\right) d t}{\left(\sqrt{t}+\mu_{Z}\right)^{2}(\sqrt{t}+\mu)} ; \quad I_{Z 1}=\int_{0}^{z_{0}} \frac{F(t) \sqrt{t} d t}{\left(\sqrt{t}+\mu_{Z}\right)^{2}(\sqrt{t}+\mu)(1-s)} \\
& I_{Z 2}=\int_{0}^{z_{0}} \frac{F^{2}(t) t d t}{\left(\sqrt{t}+\mu_{Z}\right)^{2}(\sqrt{t}+\mu)(1-s)} ; \mu=\frac{g|\lambda|}{16 \sqrt{2} \pi} ; \quad \mu_{Z}=\frac{g|\lambda|}{16 \sqrt{2} \pi(1-s)} \\
& t=\frac{G_{W}^{2}\left(p^{2}\right)^{2}}{512 \pi^{2}} ; s=\sin ^{2} \theta_{W} .
\end{aligned}
$$

We take form-factor $F(t)=F(p,-p, 0)$ from results of work [15] in which the compensation approach was applied to the electro-weak interaction:

$$
\begin{aligned}
& F(t)=\frac{1}{2} G_{15}^{31}\left(\left.t\right|_{1,1 / 2,0,-1 / 2,-1} ^{0}\right)-\frac{85 \sqrt{2} g_{0}}{128 \pi} G_{15}^{31}\left(\left.t\right|_{1,1 / 2,1 / 2,-1 / 2,-1} ^{1 / 2}\right)+C_{1} G_{04}^{10}\left(t \mid \frac{1}{2}, 1,-\frac{1}{2},-1\right)+ \\
& C_{2} G_{04}^{10}\left(t \mid 1, \frac{1}{2},-\frac{1}{2},-1\right) ; t=\frac{G_{W}^{2}\left(p^{2}\right)^{2}}{512 \pi^{2}} ; F(t)=0, t \geq t_{0} ; t_{0}=9.6175, \\
& g_{0}=0.6037, C_{1}=-0.0351, C_{2}=-0.0511 .
\end{aligned}
$$

Here $g_{0}$ is the value of gauge electro-weak coupling $g$ at point $t=t_{0}$ and we use Meijer functions

$$
G_{p q}^{m n}\left(\left.t\right|_{b_{1} \ldots, b_{q}} ^{a_{1} \ldots a_{p}}\right)
$$

for more details see, e.g. book [16]. 
With definitions $(7,8,9)$ we calculate the couplings and show results in Table 1 . Note, that additional interactions (7) were already considered e.g. in works $[17,18]$.

Table 1. Coupling constants $G_{V V^{\prime}} \mathrm{GeV}^{-2}$ of effective interactions $H V V^{\prime}$ in dependence on value of $\lambda$. All coupling values are multiplied by $10^{7}$.

\begin{tabular}{||c|c|c|c|c||}
\hline$\lambda$ & $G_{W W}$ & $G_{Z Z}$ & $G_{Z \gamma}$ & $G_{\gamma \gamma}$ \\
\hline 0.01 & 3.10 & 4.40 & 4.71 & 1.26 \\
\hline 0.006 & 2.20 & 3.10 & 3.32 & 0.89 \\
\hline 0.003 & 1.33 & 1.86 & 2.00 & 0.54 \\
\hline 0 & 0 & 0 & 0 & 0 \\
\hline-0.003 & -3.51 & -4.83 & -5.17 & -2.58 \\
\hline-0.006 & -6.54 & -9.01 & -9.65 & -2.58 \\
\hline-0.01 & -10.3 & -14.2 & -15.2 & -4.08 \\
\hline \hline
\end{tabular}

Then we calculate cross sections of pair weak boson production accompanied by the Higgs. In doing this we apply the CompHEP package [19]. In subsequent Tables we show results for values of couplings in (7) and cross sections of processes $p+p \rightarrow W^{+} W^{-} H+X(\sigma(+-)), p+p \rightarrow W^{+} Z H+X$ $(\sigma(+0))$ and $p+p \rightarrow W^{-} Z H+X(\sigma(-0))$. Results are shown in dependence on value of $\lambda$ in admissible interval (3).

Table 2. Production LO cross sections of $V V^{\prime} H$ for $\sqrt{s}=8 \mathrm{TeV}$ at the LHC.

\begin{tabular}{||c|c|c|c||}
\hline$\lambda$ & $\sigma(+-) f b$ & $\sigma(+0) f b$ & $\sigma(-0) f b$ \\
\hline-0.01 & 5.18 & 1.21 & 0.499 \\
\hline-0.006 & 4.62 & 1.09 & 0.45 \\
\hline-0.003 & 4.22 & 1.02 & 0.43 \\
\hline 0 & 3.86 & 0.98 & 0.42 \\
\hline 0.003 & 3.75 & 0.98 & 0.42 \\
\hline 0.006 & 3.69 & 0.98 & 0.42 \\
\hline 0.01 & 3.62 & 0.98 & 0.42 \\
\hline \hline
\end{tabular}

Table 3. Production LO cross sections of $V V^{\prime} H$ for $\sqrt{s}=13 \mathrm{TeV}$ at the LHC.

\begin{tabular}{||c|c|c|c||}
\hline$\lambda$ & $\sigma(+-) f b$ & $\sigma(+0) f b$ & $\sigma(-0) f b$ \\
\hline-0.01 & 21.16 & 3.60 & 1.47 \\
\hline-0.006 & 17.06 & 2.71 & 1.20 \\
\hline-0.003 & 14.36 & 2.27 & 1.07 \\
\hline 0 & 11.90 & 2.08 & 1.00 \\
\hline 0.003 & 11.14 & 2.09 & 1.00 \\
\hline 0.006 & 10.70 & 2.12 & 1.02 \\
\hline 0.01 & 10.35 & 2.19 & 1.03 \\
\hline \hline
\end{tabular}

From Table 3 we see, that for $\sqrt{s}=13 \mathrm{TeV}$ with negative $\lambda$ the effect is noticable, especially for process $p+p \rightarrow W^{+} W^{-} H+X$, and $e . g$ for $\lambda=-0.01$ the cross-section is almost two times more than the SM one. However the cross-section itself is presumably not sufficiently high for a productive study of the effect. 
Let us note also, that $V V^{\prime} H$ the additional interaction with $\lambda \neq 0$ might give effect for VBF Higgs production. However calculations show, that with couplings from Table 1 effects even for $\sqrt{s}=13 \mathrm{TeV}$ are insignificant, as well as effects for branching ratios of the Higgs decays.

We have also calculated effects of the interaction for an associated single electro-weak boson production with the Higgs. While cross sections of processes are significant (few hundreds of $f b$ ), contributions of the additional interaction do not exceed few per cent. For example, for process $p p \rightarrow W^{+} H+X$ at $\sqrt{s}=13 \mathrm{TeV}$ with $|\lambda|=0.01$ ratio $\mu=1.033$.

Possible manifestations of vertices (7) were studied in decays $H \rightarrow W^{+} W^{-}, H \rightarrow Z Z$ [18] . Results of this work give limitations, which definitely do not contradict values for couplings presented in Table 1.

In the next section we consider additional contributions of interactions (7) to interaction of the Higgs with quarks, especially with the heavy ones, which can lead to essentially more significant effects at the LHC.

\section{Additional top and bottom quarks interaction with the Higgs}

We use vertices (6) to define additional contribution for quarks interactions with Higgs. For the beginning we shall be interested in interactions of the most heavy top quarks.

Taking into account these vertices we calculate loop diagrams presented in Fig. 2 to obtain the following expression for $\bar{t} t H$ vertex, which corresponds to the first diagram in Fig.2

$$
V_{\bar{t} t H}=-\frac{g}{2 M_{W}} \bar{t}\left(M_{t}+9 \cos \theta_{W} M_{Z} M_{W} G_{W W} I_{1}\left(\hat{p}_{1}-\hat{p}_{2}\right)\left(1+\gamma_{5}\right)\right) t H ; \hat{a}=a_{\mu} \gamma^{\mu} ;
$$

where $G_{W W}$ is already defined in (6) and calculated in Table $1, p_{1}$ and $p_{2}$ are respectively outcoming momenta of $t$ and $\bar{t}$ quarks. Integral $I_{1}$ is defined in (8). For calculation of the integral we here use the same form-factor $F(t)(9)$.

Due to QCD gauge invariance we have to take into account also vertex for fourfold interaction involving also a gluon: $\bar{t} t H G_{\mu}$, which actually corresponds to the second diagram in Fig.2

$$
V_{\bar{t} t H G}=9 g g_{s} \cos \theta_{W} M_{Z} G_{W W} I_{1} \bar{t} \hat{G}\left(1+\gamma_{5}\right) t H
$$

where $g_{s}$ is the QCD gauge coupling constant and of course usual structure of the QCD is used. Then we perform calculations for cross sections of process $p+p \rightarrow \bar{t} t H+X$ for two energies of the LHC: $\sqrt{s}=8 \mathrm{TeV}$ and $\sqrt{s}=13 \mathrm{TeV}$

Let us define for the same values of $\sqrt{s}$ ratios of cross-sections with nonzero $\lambda$ in admissible interval (3) and its SM value for $\lambda=0$

$$
\mu_{\sqrt{s}}=\frac{\sigma_{\lambda}(p p \rightarrow \bar{t} t H)}{\sigma_{0}(p p \rightarrow \bar{t} t H)}
$$

where $\sigma_{0}$ is actually $\mathrm{SM}$ value for the cross section. Results of calculations with application of CompHEP package [19] are shown in Table 4. LHC.

Table 4. Production LO cross sections $f b$ of $\bar{t} t H$ for $\sqrt{s}=8 \mathrm{TeV}, 13 \mathrm{TeV}$ and ratio $\mu_{\sqrt{s}}$ (12) at the 


\begin{tabular}{||c|c|c|c|c||}
\hline$\lambda$ & $\sigma(13 \mathrm{TeV})$ & $\sigma(8 \mathrm{TeV})$ & $\mu_{13}$ & $\mu_{8}$ \\
\hline-0.01 & 1628.2 & 460.6 & 3.14 & 3.15 \\
\hline-0.006 & 1212.9 & 342.6 & 2.34 & 2.34 \\
\hline-0.003 & 853.3 & 241.0 & 1.65 & 1.65 \\
\hline 0 & 517.8 & 146.1 & 1.00 & 1.00 \\
\hline 0.003 & 401.4 & 113.6 & 0.78 & 0.78 \\
\hline 0.006 & 348.7 & 98.4 & 0.67 & 0.67 \\
\hline 0.01 & 304.4 & 85.9 & 0.59 & 0.59 \\
\hline \hline
\end{tabular}

Values for cross-sections are calculated with the current value for the strong coupling [20]

$$
\alpha_{s}\left(M_{Z}\right)=0.1181 \pm 0.0011
$$

The uncertainty in (13) means $2 \%$ accuracy for calculated ross-sections. With taking into account of other sources of uncertainties we estimate overall accuracy to be around $10 \%$.

The combination of the ATLAS and the CMS data, collected with $\sqrt{\mathrm{s}}=7 \mathrm{TeV}$ and $8 \mathrm{TeV}$, gives the following experimental result for ratio $\mu_{8}[21]$

$$
\mu_{8}=2.3_{-0.6}^{+0.7}
$$

Due to significant uncertainties, result (14) does not mean convincing deviation from the SM value. With numbers from Table 4 we have from (14) the following prediction

$$
\lambda=-0.0057_{-0.0039}^{+0.0028} \text {. }
$$

The result is safely inside experimental limitations $(3,4)$. Let us note, that estimates for cut-off an energy scale also evidently do not contradict LHC results [9]. Of course we have in (15) again only two standard deviations effect, which undoubtedly needs further studies. We see that values of $\mu$, practically, do not depend on $\sqrt{s}$ but with $\sqrt{s}=13 \mathrm{TeV}$ cross sections are more than three times as much as those for conditions of result (14). One might hope to check the predictions in forthcoming experimental studies at the LHC with increased statistics. Emphasize, that in case of this study would give result $\lambda \neq 0$, we would come to the fundamental conclusion of non-perturbative effects in the electro-weak interaction to be necessarily present. Let us note, that recent NLO and NNLL SM calculations of $\bar{t} t H$ production cross section at $13 \mathrm{TeV}$ are presented in works [22-24].

Let us consider also associative production of the Higgs with $\bar{b} b$ pairs. Unlike the $t$ quark pairs case, for which the experimental studies were performed and have given results, $e$. $g$. (14), there were no dedicated studies. However, interactions $(10,11)$ in our consideration also exist for other quarks. All the difference is connected only with value of the quark mass in (10). In particular, it is advisable to consider also process of $b$ quark associative pair production with Higgs

$$
p+p \rightarrow \bar{b} b H+X
$$

Results of calculations are shown in Table 5. LHC.

Table 5. Production LO cross sections $f b$ of $\bar{b} b H$ for $\sqrt{s}=8 \mathrm{TeV}, 13 \mathrm{TeV}$ and ratio $\mu_{b \sqrt{s}}$ at the 


\begin{tabular}{||c|c|c|c|c||}
\hline$\lambda$ & $\sigma(13 \mathrm{TeV})$ & $\sigma(8 \mathrm{TeV})$ & $\mu_{b 13}$ & $\mu_{b 8}$ \\
\hline-0.01 & 1612.5 & 572.7 & 2.93 & 2.77 \\
\hline-0.006 & 1204.4 & 433.6 & 2.19 & 2.10 \\
\hline-0.003 & 903.0 & 329.1 & 1.64 & 1.59 \\
\hline 0 & 550.8 & 206.7 & 1.00 & 1.00 \\
\hline 0.003 & 446.0 & 151.8 & 0.81 & 0.82 \\
\hline 0.006 & 396.4 & 151.8 & 0.72 & 0.73 \\
\hline 0.01 & 354.6 & 137.1 & 0.64 & 0.66 \\
\hline \hline
\end{tabular}

Effects for the b-quarks are of the same order of magnitude as for the $t$-quark pairs. Unlike the $t$-quark case we have no datum for a comparison. As a matter of fact, analogous results are valid for light quarks $u, d, c, s$ as well.

\section{Conclusion}

The problem of an existence of non-perturbative contributions in the electro-weak interaction is without doubt a fundamental one. Anomalous three-boson interaction (2) provides the crucial test for this problem. We have shown above, that there are promising processes

$$
p+p \rightarrow \bar{t} t H+X \quad p+p \rightarrow \bar{b} b H+X
$$

for investigation of the problem at the LHC, and we can hope, that future results for these processes at $\sqrt{s}=13 \mathrm{TeV}$ will confirm the existence of non-perturbative effects in the electro-weak interaction. Let us note, that we have studied how results for processes under the study depend on different cuts. It comes out, that for main process $p+p \rightarrow \bar{t} t H$ cuts $M(\bar{t} t)>M_{0}, M(t H)>M_{0}^{\prime}, p_{T}(H)>p_{T 0}$ etc lead, of course, to decreasing of cross sections, but, practically, do not change ratios $\mu$ for data. Thus introduction of cuts is to be defined by conditions of experiments, in particular by background considerations.

The important problem is, if predictions under the discussion could in any way contradict the present knowledge. We have already mentioned, that contributions of the additional interactions to branching ratios of the Higgs are negligible. The effects in process $p+p \rightarrow \bar{q} q H+X$, where we have to take into account all six flavors of quarks would lead to an additional contribution to the total Higgs production cross section. For example, for $\lambda=-0.006$, which actually is quite close to the central value in estimate (15) we have from Tables 4, 5 the following additional contributions $\Delta \sigma$ to the total cross section of the Higgs production

$$
\begin{aligned}
& \Delta \sigma(8 T e V)=1.36 p b ; \quad \sigma(8 T e V)=22.3 p b ; \\
& \Delta \sigma(13 T e V)=4.03 p b ; \sigma(13 T e V)=50.6 p b ;
\end{aligned}
$$

where we also show the SM values for the total cross-sections [20]. These additional contributions lead to a change in the global signal strength, which currently reads [20]

$$
\mu=1.09 \pm 0.07 \pm 0.04 \pm 0.03 \pm 0.07
$$

where two last errors are connected with uncertainties in the theoretical estimates. We easily see, that additional contributions (18) give the following changes for theoretical predictions for effective $\mu$ instead of unity

$$
\mu(8 T e V)=1.061 ; \quad \mu(13 T e V)=1.080 .
$$


The results evidently do not contradict to value (19), which is based mostly on data collected with $\sqrt{s}=7$ and $8 \mathrm{TeV}$.

In case of an existence of triple interaction (2), e.g. in processes (17), being confirmed, extensive studies of other possible non-perturbative effects will be desirable. Let us emphasize that simultaneous consideration of effects in reaction (17) and searches for anomalous interaction (2) are of the utmost importance. Provided these searches would give a positive and compatible results, we would obtain the strong evidence in favour of non-perturbative contributions of the electro-weak interaction.

Let us note also, that effects in top pair production in association with an electro-weak boson $W^{ \pm}, Z$, which were discussed in work [25] under assumption of wouldbe existence of four-fold electroweak bosons effective interaction [26, 27]. These effects might also indicate for the non-perturbative contributions.

In case the non-perturbative effects being discussed above would be discovered, there could appear new and really attractive possibilities. The approach $[13-16,25,28]$ is connected with non-trivial solutions of compensation equations. Such solutions exist only for definite values of parameters inherent in a problem under a study. For example, the non-trivial solutions exist for values of coupling constants at the characteristic scale $\Lambda$ for QCD $g_{s}\left(\Lambda_{s}\right)=3.116$ [14] and for the EW theory $g_{E W}\left(\Lambda_{E W}\right)=0.604$ [15] (see also (9)), that seems quite reasonable. Thus in case of the successful confirmation of the approach, we could hope to find a way for understanding of why physical values of couplings and maybe also of mass ratios are such as we do observe.

\section{Acknowledgment}

The author appreciates the collaboration with I.V. Zaitsev in obtaining results of the talk (see works $[25,28])$. The studies are supported in part by grant NSh-7989.2016.2 of the President of Russian Federation. 

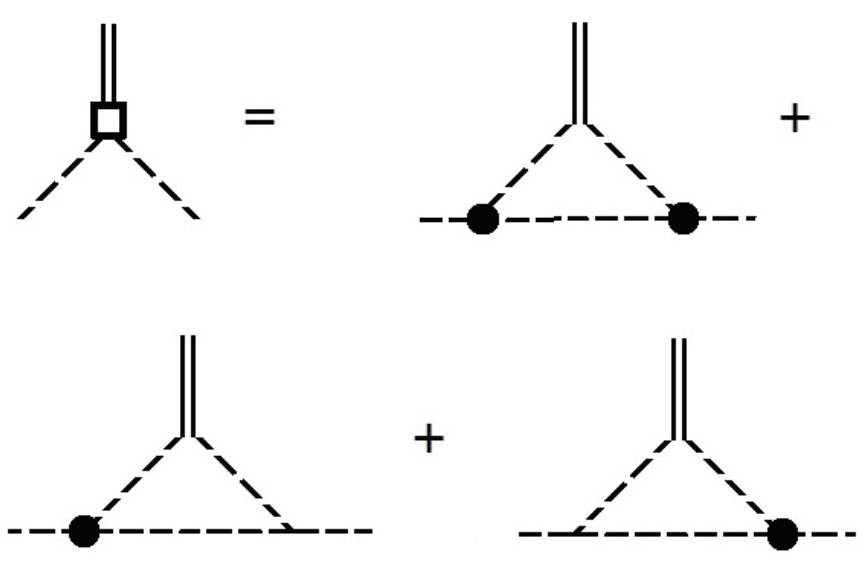

Figure 1. Diagram representation of non-perturbative contribution to $V V H$ vertices depicted as square. Double lines represent Higgs boson, dotted lines represent electro-weak bosons. Black spots represent non-perturbative vertex $(2,6)$ and simple points corresponds to usual SM interaction.
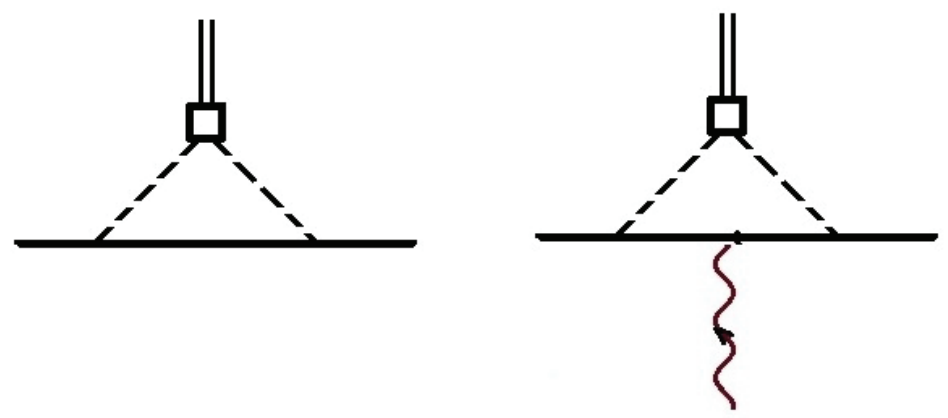

Figure 2. Diagram representation of non-perturbative contribution to $\bar{t} t H$ and $\bar{t} t H G$. Double lines represent Higgs boson, dotted lines represent electro-weak bosons. Thick lines represents the $t$ quark, the wavy line corresponds to a gluon. Non-perturbative part of $V V H$ vertex is defined in the previous section and simple points correspond to usual gauge interaction.

\section{References}

[1] Y. Nambu and G. Jona-Lasinio, Phys. Rev., 122 (1961), p. 345.

[2] Y. Nambu and G. Jona-Lasinio, Phys. Rev., 124 (1961), p. 246.

[3] M. K. Volkov, Annals Phys.,157 (1984), p. 282.

[4] D. Ebert, H. Reinhardt and M. K. Volkov, Prog. Part. Nucl. Phys., 33 (1994), p. 1.

[5] M. K. Volkov and A. E. Radzhabov, Phys. Usp., 49 (2006), p. 551. 
[6] A. I. Alekseev, B. A. Arbuzov and V. A. Baikov, Theor. Math. Phys., 52 (1982), p. 739.

[7] K. Hagiwara, R. D. Peccei, D. Zeppenfeld and K. Hikasa, Nucl. Phys. B, 282 (1987), p. 253.

[8] K. Hagiwara, S. Ishihara, R. Szalapski and D. Zeppenfeld, Phys. Rev. D, 48 (1993), p. 2182.

[9] A.M. Sirunyan et al. (CMS Collaboration), Phys. Lett. B, 772 (2017) p. 21.

[10] M. Aabud et al. (ATLAS Collaboration), Eur. Phys. J. C, 77 (2017) p. 563.

[11] N. N. Bogoliubov, Soviet Phys.-Uspekhi, 67 (1959), p. 236.

[12] N. N. Bogoliubov, Physica Suppl. (Amsterdam), 26 (1960), p. 1.

[13] B. A. Arbuzov, M. K. Volkov and I. V. Zaitsev, Int. J. Mod. Phys. A, 21 (2006), p. 5721.

[14] B. A. Arbuzov and I. V. Zaitsev, Int. J. Mod. Phys. A, 28 (2013), Article 1350127.

[15] B. A. Arbuzov and I. V. Zaitsev, Phys. Rev. D, 85 (2012), Article 093001.

[16] B. A. Arbuzov, Non-perturbative Effective Interactions in the Standard Model, De Gruyter, Berlin, 2014.

[17] I. Anderson et al., Phys. Rev. D, 89 (2014), Article 035007.

[18] V. Khachatryan et al. (CMS Collaboration) Phys.Rev. D, 92 (2015) Article 012004.

[19] E. E. Boos et al. (CompHEP Collaboration), Nucl. Instr. Meth. A, 534 (2004), p. 250.

[20] C. Patringnani et al. (Particle Data Group), Chin. Phys. C, 40 (2016), Article 100001.

[21] G. Aad et al. (ATLAS and CMS Collaborations), J. High Energy Phys., 1608 (2016), p. 045.

[22] A. Broggio et al., JHEP, 1603 (2016) p. 124.

[23] A. Broggio et al., JHEP, 1702 (2017) p. 126.

[24] A. Kulesza et al., arXiv: 1704.03363 [hep-ph].

[25] B. A. Arbuzov and I. V. Zaitsev, Prog. Theor. Exp. Phys. 85 (2016), Article 093001.

[26] G. Belanger and F. Boudjema, Phys. Lett. B, 288 (1992), p. 201.

[27] G. Belanger et al., Eur. Phys. J. C, 13 (2000), p. 283.

[28] B. A. Arbuzov and I. V. Zaitsev, Phys. Lett. B, 772 (2017), p. 184. 